\title{
Genotypes and Characteristics of Phenamacril-Resistant Mutants in Fusarium asiaticum
}

Bin Li, Zhitian Zheng, Xiumei Liu, Yiqiang Cai, Xuewei Mao, and Mingguo Zhou, College of Plant Protection, Nanjing Agricultural University, Key Laboratory of Pesticide, Nanjing, Jiangsu Province, 210095, China

\begin{abstract}
Li, B., Zheng, Z., Liu, X., Cai, Y., Mao, X., and Zhou, M. 2016. Genotypes and characteristics of phenamacril-resistant mutants in Fusarium asiaticum. Plant Dis. 100:1754-1761.

Fusarium asiaticum is a critical pathogen of Fusarium head blight (FHB) in the southern part of China. The fungicide phenamacril has been extensively used for controlling FHB in recent years, which reduced both FHB severity and mycotoxin production. Our previous report indicated that resistance of $F$. asiaticum to phenamacril was related to mutations in myosin5. A recent article revealed that the resistance level of phenamacril-resistant mutants was associated with the genotypes of myosin 5 in these mutants. In total, we obtained 239 resistant isolates by fungicide domestication, and 82 resistant mutants were randomly selected for further study. Of these mutants, 25.6, 7.3, and 67.1\% showed low resistance (LR), moderate resistance (MR), and high resistance (HR), respectively, to phenamacril determined

by $50 \%$ effective concentration values. Point mutations A135T, V151M, P204S, I434M, A577T, R580G/H, or I581F led to LR. Point mutations S418R, I424R, and A577G were responsible for MR and point mutations K216R/E, S217P/L, or E420K/G/D conferred HR. Interestingly, all of the mutations concentrated in the myosin 5 motor domain and mutations conferring HR occurred at codon 217 and 420, which we called the core region. Homology modeling revealed that mutations far from the core region led to a lower resistance degree. Phenotype assays revealed that the most highly resistant mutants did not significantly change pathogenicity but decreased conidia production compared with the wild type, which may slow down the formation of the resistant pathogen population in the fields.
\end{abstract}

Fusarium head blight (FHB) of wheat and other small cereal grains is a devastating disease worldwide, which can produce harmful mycotoxins in infected grain and threaten animal and human health (Bai and Shaner 2004; Bottalico 1998; Goswami and Kistler 2004). Thus far, 11 phylogenetic species are causal agents of FHB and these species belong to the Fusarium graminearum Schwabe species complex of $\beta$-trichothecene toxin producers (O'Donnell et al. 2004; Starkey et al. 2007; Suga et al. 2008; Tóth et al. 2005). FHB disease has become increasingly severe in the middle and lower reaches of the Yangtze River, the southern winter wheat region, and the northeastern spring wheat region in China since FHB was first reported in 1936 (Chen et al. 2000). Zhang et al. (2007) reported that $77.3 \%$ of the 299 isolates collected from wheat of various regions that experienced FHB epidemics in China were identified as $F$. asiaticum, and another 68 isolates were confirmed to be $F$. graminearum.

Because few wheat cultivars are available with natural resistance to Fusarium spp., the control of FHB has depended on application of fungicides during wheat anthesis in the past few decades (Chen et al. 2011; Parry et al. 1995; Windels 2000). A novel cyanoacrylate fungicide, phenamacril (JS399-19), has been extensively used to control FHB and reduce mycotoxin contamination, such as $25 \%$ SC (certificate number LS20072657). (Chen and Zhou 2009; Diao et al. 2012; Yu et al. 2011; Zhang et al. 2010b). However, mutants with levels of resistance to phenamacril have been easily obtained by fungicide domestication and UV irradiation in F. asiaticum (Chen et al. 2008). We also found that point mutations of myosin5 were responsible for phenamacril resistance in $F$. graminearum through comparison of the myosin5 gene sequences between wild-type and highly resistant mutants (Zheng et al. 2015).

In addition, Zhang et al. (2015) revealed that JS399-19 (phenamacril) inhibited ATPase activity of the FgMyo1 (myosin5) motor domain and had a serious effect on localization of FgMyo1 at the tips of germlings.

Corresponding author: M. Zhou; E-mail: mgzhou@njau.edu.cn

Accepted for publication 19 April 2016.

http://dx.doi.org/10.1094/PDIS-02-16-0169-RE

(C) 2016 The American Phytopathological Society
Myosin5 is actin-dependent ATPase motor and contains two motor domains: tail homology 1 and src homology 3 . In $F$. graminearum, myosin 5 encodes an essential gene and the myosin 5 deletion mutant had a lethal effect on this fungus (Zhang et al. 2015).

It is well known that mutations at different drug target sites generate variable degrees of drug resistance. For example, a change at codon 198 in $\beta 2$-tubulin of $F$. asiaticum will confer high resistance to benzimidazoles, and a change at codon 167 will confer moderate resistance (Qiu et al. 2011). In this article, we studied the frequency of low, moderate, and high resistance to phenamacril in $F$. asiaticum mutants, and evaluated their fitness with the biological characteristics of growth rate, colony morphology, and asexual reproduction. Genotypes associated with resistance degree were also analyzed by sequencing the whole myosin 5 gene. These results will help to understand the risk of phenamacril resistance and develop molecular techniques to diagnose phenamacril resistance in F. asiaticum.

\section{Materials and Methods}

Fungicide and media. Technical-grade phenamacril (95\%; experimental code JS399-19; active ingredient 2-cyano-3-amino-3-phenylancryic acetate) was provided by Jiangsu Branch of the National Pesticide Research \& Development South Center and dissolved in methanol to 1 and $4 \mathrm{mg} / \mathrm{ml}$ for stock solution, which was added into autoclaved media for testing inhibition of mycelia growth. Potato dextrose agar (PDA; $200 \mathrm{~g}$ of potato, $20 \mathrm{~g}$ of glucose, $15 \mathrm{~g}$ of agar, and 1 liter of water) was used for routine cultures and for testing sensitivity of phenamacril. Mung bean liquid (MBL) medium (30 $\mathrm{g}$ of mung bean boiled in 1 liter of water for $20 \mathrm{~min}$; the mixture was then filtered through cheesecloth) was prepared for conidia production. Water agar (2\% agar) medium was used to recover single-spore isolates.

Recovery of phenamacril-resistant mutants. In all, 1,000 fresh mycelial plugs from the colony margins of sensitive strain 2021 were transferred to the 250 PDA plates amended with phenamacril at $10 \mu \mathrm{g} / \mathrm{ml}$ and incubated in a growth chamber at $25^{\circ} \mathrm{C}$. Any spontaneous and fast-growing sectors from the restricted colonies on PDA plates were selected for resistance identification on PDA plates amended with phenamacril at $1.5,15$, or $75 \mu \mathrm{g} / \mathrm{ml}$. For the stable phenamacril-resistant mutants, single-spore isolates were established. The number of resistant mutants was counted (Chen et al. 2008). 
Stability of the resistance to phenamacril. All the resistant mutants and their parental $F$. asiaticum strain 2021 were transferred eight times onto new PDA plates with no fungicide, by transferring single mycelial plugs to the new PDA plates every 5 days for a period of 40 days, or stored at $4{ }^{\circ} \mathrm{C}$ in the refrigerator for 40 days. Resistance to phenamacril was determined again for resistance stability.

Characterization of $\boldsymbol{F}$. asiaticum mutants resistant to phenamacril. For all of these $F$. asiaticum mutants, the levels of resistance to phenamacril were determined by transferring $5-\mathrm{mm}$ plugs from the edge of an actively growing colony (3 days old) to PDA media amended with phenamacril at $0,1.5,15$, and $75 \mu \mathrm{g} / \mathrm{ml}$ simultaneously; plugs were incubated at $25^{\circ} \mathrm{C}$ in the growth chamber for 3 to 5 days. Then, we randomly selected 82 mutants from among the 239 mutants to narrow the sample. Based on the colony growth of the 82 mutants at various concentrations of phenamacril $(0,1.5,15$, and $75 \mu \mathrm{g} / \mathrm{ml}$ ), we placed the mycelial plugs ( $5 \mathrm{~mm}$ in diameter) which grew at 1.5 to 15,15 to 75 , and $>75 \mu \mathrm{g} / \mathrm{ml}$ on the center of a PDA plate amended with JS399-19 at $0,1,2,4$, or $8 \mu \mathrm{g} / \mathrm{ml} ; 0,10,20$, 40, or $80 \mu \mathrm{g} / \mathrm{ml}$; or $0,50,100,200$, or $400 \mu \mathrm{g} / \mathrm{ml}$, respectively. There were three replicates for each concentration. After cultures were kept at $25^{\circ} \mathrm{C}$ for 3 to 14 days, the $50 \%$ effective concentration $\left(\mathrm{EC}_{50}\right)$ values of resistant mutants were calculated by regressing percent growth inhibition against the log of fungicide concentration with DPS software, as described previously ( $\mathrm{Li}$ et al. 2008). According to mean $\mathrm{EC}_{50}$ values, these 82 resistant mutants were divided into groups showing low resistance (LR, 1.5 to $15 \mu \mathrm{g} / \mathrm{ml}$ ), moderate resistance (MR, 15.1 to $75 \mu \mathrm{g} / \mathrm{ml}$ ), and high resistance (HR, $>75 \mu \mathrm{g} / \mathrm{ml}$ ), determined by $\mathrm{EC}_{50}$ values as in the previous classified method (Chen et al. 2008).

Hyphal growth and conidia production of $F$. asiaticum mutants resistant to phenamacril. After culturing these mutants for 3 days on PDA, mycelial plugs ( $5 \mathrm{~mm}$ in diameter) of the mutants were transferred to PDA plates at $25^{\circ} \mathrm{C}$ for 3 days. Three replicates were performed for each of the selected mutants and the tests were repeated twice. Colony diameter (in centimeters) was measured to compare mycelial linear growth, and means were compared with Fisher's least significant difference test with DPS software. Photographs of colony morphology were taken on the third day.

For conidium production assays, 10 mycelial plugs $(5 \mathrm{~mm}$ in diameter), taken from the periphery of a 3-day-old colony of randomly selected resistant mutants, were added to a 200-ml flask containing $100 \mathrm{ml}$ of MBL medium. Each strain was represented by three flasks. The flasks were incubated at $25^{\circ} \mathrm{C}$ for 7 days with shaking $(185 \mathrm{rpm})$. Production of conidia was assessed after 7 days of incubation using a hemocytometer and a microscope. The experiment was conducted three times.

DNA extraction. The selected mutants were cultured on PDA for 5 to 14 days and aerial hyphal were obtained for DNA extraction. The aerial hyphal were suspended in $700 \mathrm{ml}$ of cetyltrimethylammonium bromide (100 mM Tris- $\mathrm{HCl}$ [pH 8.0], $20 \mathrm{mM} \mathrm{EDTA}$, and $1.5 \mathrm{M} \mathrm{NaCl}$ ) and $700 \mathrm{ml}$ of phenol + chloroform + isoamyl alcohol (25:24:1). After the suspensions had been incubated at $37^{\circ} \mathrm{C}$ for $2 \mathrm{~h}$, the aqueous layers were extracted once with chloroform, and isopropyl alcohol was added to precipitate DNA. The DNA pellet was collected by centrifugation, washed with $70 \%$ ethanol, and resuspended after drying in $50 \mu \mathrm{l}$ of Millipore water (Brandfass and Karlovsky 2008).

Cloning and sequencing of myosin5 gene in phenamacrilresistant isolates of $\boldsymbol{F}$. asiaticum. Complete DNA sequences of a myosin 5 locus (ranging from $313 \mathrm{bp}$ upstream to $202 \mathrm{bp}$ downstream of the myosin 5 coding region) in $F$. asiaticum were obtained using a BLAST search of the Fusarium genome (http://www.broadinstitute. org/annotation/genome/fusarium_group/MultiHome.html). These sequences were used to design the polymerase chain reaction (PCR) primer pairs 01410F 5'-TCCCGTCAGGCACCACTA-3' and 01410R 5'-CGGTTGCCCAGTTCAGTC-3', that were used for amplification of the whole fragment of the myosin5 gene (FGSG 01410). PCR mixtures contained total DNA $(25 \mathrm{ng} / \mu \mathrm{l}), 1.5 \mathrm{mM} \mathrm{MgCl} 2,200 \mu \mathrm{M}$ $\mathrm{dNTP}$, and $2.5 \mathrm{U}$ of LA Taq DNA polymerase. PCR was performed using a TaKaRa PCR Thermal Cycler Dice. The PCR program included an initial denaturation step at $94^{\circ} \mathrm{C}$ for $5 \mathrm{~min}$; followed by 35 cycles at $94^{\circ} \mathrm{C}$ for $30 \mathrm{~s}, 53^{\circ} \mathrm{C}$ for $1 \mathrm{~min}$, and $72^{\circ} \mathrm{C}$ for $4 \mathrm{~min}$; a final extension for $10 \mathrm{~min}$ at $72^{\circ} \mathrm{C}$; and holding at $12^{\circ} \mathrm{C}$. The amplified myosin 5 gene, of approximately $3.8 \mathrm{~kb}$ in size (eliminated upstream 313 bp and downstream 202 bp of the myosin 5 coding region), was obtained from genomic DNA of $F$. asiaticum. Individual amplified fragments were purified, cloned, and then sequenced and used to compare the myosin 5 sequence between wild-type strain 2021 and resistant mutants using V7.0.9.0 Bioedit (Isis Pharmaceuticals) (Zheng et al. 2015).

The virulence assays of mutants with different genotypes. Seed of 'Zhenmai' wheat were incubated on moist filter paper at $20^{\circ} \mathrm{C}$ for 3 days and used to assay mutant virulence (Wu et al. 2005). The top 2 to $3 \mathrm{~mm}$ of the coleoptiles were cut from the 3-day-old seed, and the tip was injected with $10 \mu \mathrm{l}$ of conidial suspension. The coleoptiles were then incubated at $25^{\circ} \mathrm{C}, 95 \%$ relative humidity, and $12 \mathrm{~h}$ of fluorescent light per $24 \mathrm{~h}$. There were 10 coleoptiles per isolate and three repeat experiments to ensure the accuracy the assays. The pathogenicity of the strains and mutants were assessed by measuring the length of brown lesions on diseased stems at 14 days postinoculation (dpi) (Zhang et al. 2010a).

Homology modeling of the $F$. asiaticum myosin5 motor domain. The sequence alignment of the myosin 5 motor domain displayed $46 \%$ identity with the crystal structure of the actin-tropomyosin-myosin complex in Dictyostelium discoideum (PDBID:4A7F), which was used to perform the homology modeling on the Discovery Studio 3.5. A three-dimensional model of the $F$. graminearum myosin 5 motor domain was built. The resulting structure was viewed with the $\mathrm{Cn} 3 \mathrm{D}$ 4.3.1 three-dimensional structure viewer.

\section{Results}

Induction of phenamacril-resistant mutants from phenamacrilsensitive isolate 2021. All 1,000 fresh mycelial plugs from phenamacrilsensitive strain 2021 grew poorly on PDA plates amended with phenamacril at $10 \mu \mathrm{g} / \mathrm{ml}$ during the first 10 days. Only a few spontaneous sectors of these plugs grew fast after 15 days, and 239 resistant mutants from the growing sectors were obtained by selection for resistance to the fungicide after 20 days. However, their parental strain 2021 cannot grow any mycelia. The 239 resistant mutants, which were recovered from the phenamacril-sensitive strain 2021, grew well on the PDA plates amended with phenamacril at $1.5,15$, or $75 \mu \mathrm{g} / \mathrm{ml}$.

Resistance stability of the resistant mutants. All of the 239 single-spore progenies of each resistant mutant grew normally on PDA plates amended with phenamacril at $1.5,15$, or $75 \mu \mathrm{g} / \mathrm{ml}$, indicating stability of resistance to this fungicide. Based on colony growth on PDA plates amended with phenamacril at $1.5,15$, or $75 \mu \mathrm{g} / \mathrm{ml}$, all of the resistant mutants maintained their resistance to phenamacril through eight transfers on PDA plates for 40 days, and when stored on PDA slants at $4{ }^{\circ} \mathrm{C}$ for 40 days (Fig. 1).

Frequency of different resistance levels of $F$. asiaticum mutants to phenamacril. After testing the $\mathrm{EC}_{50}$ of all 82 resistant mutants, we found that 21 mutants belonged to category LR, 6 mutants belonged to MR, and 55 mutants belonged to HR. Therefore, the frequency of LR, MR, and HR was $25.6,7.3$, and $67.1 \%$, respectively (Table 1). This small sample could indirectly reflect the frequency of the big sample (239 mutants) or the actual laboratory and fields sample.

Genotypes of myosin5 gene in the laboratory induced phenamacrilresistant $\boldsymbol{F}$. asiaticum mutants. Comparison of the nucleotide sequence of the myosin 5 gene in $F$. asiaticum strain 2021 revealed that 12 codon positions of point mutations (codon 135, 151, 204, 216, $217,418,420,424,434,577,580$, and 581) were present in the sequences of all the resistant mutants. Among these mutants, changes at codons $135(\mathrm{~A} \rightarrow \mathrm{T}), 151(\mathrm{~V} \rightarrow \mathrm{M}), 204(\mathrm{P} \rightarrow \mathrm{S}), 434(\mathrm{I} \rightarrow \mathrm{M}), 577$ $(\mathrm{A} \rightarrow \mathrm{T}), 580(\mathrm{R} \rightarrow \mathrm{G}, \mathrm{R} \rightarrow \mathrm{H})$, and $581(\mathrm{I} \rightarrow \mathrm{F})$ were found in $21 \mathrm{LR}$ mutants out of the 82 phenamacril-resistant mutants. Six MR mutants expressed three kinds of point mutations in the myosin5 gene, including codons $418(\mathrm{~S} \rightarrow \mathrm{R}), 424(\mathrm{I} \rightarrow \mathrm{R})$, and $577(\mathrm{~A} \rightarrow \mathrm{G})$. However, 55 resistant mutants belonged to $\mathrm{HR}$ mutants and point mutations occurred at codons $216(\mathrm{~K} \rightarrow \mathrm{R}, \mathrm{K} \rightarrow \mathrm{E}), 217(\mathrm{~S} \rightarrow \mathrm{P}, \mathrm{S} \rightarrow \mathrm{L})$, and 420 $(\mathrm{E} \rightarrow \mathrm{K}, \mathrm{E} \rightarrow \mathrm{G}, \mathrm{E} \rightarrow \mathrm{D})$ (Table 2). Among all of the point mutation types, mutation type $\mathrm{E} \rightarrow \mathrm{K}$ showed extremely high resistance (Table 2). It was noticeable that the resistant mutants with differential resistance levels (LR, MR, and HR) exhibited different point mutations in the myosin5 gene at codons 135, 151, 204, 216, 217, 418, $420,424,434,577,580$, and 581 . In addition, all of these mutations 
concentrated in the myosin5 motor domain and, as is common, two kinds of mutation positions occurred at codon 217 and 420 .

Hyphal growth rate, colony morphology, and conidia production of $F$. asiaticum mutants resistant to phenamacril. Compared with phenamacril-sensitive $F$. asiaticum strain 2021, many resistant mutants did not show any changes in mycelial growth rate on fungicide-free PDA plates, and only a few mutants exhibited growth defects, indicating no significant reduction of this fitness parameter due to resistance. However, many resistant mutants had a distinctive colony morphology on PDA plates. Mutants with mutations at codon

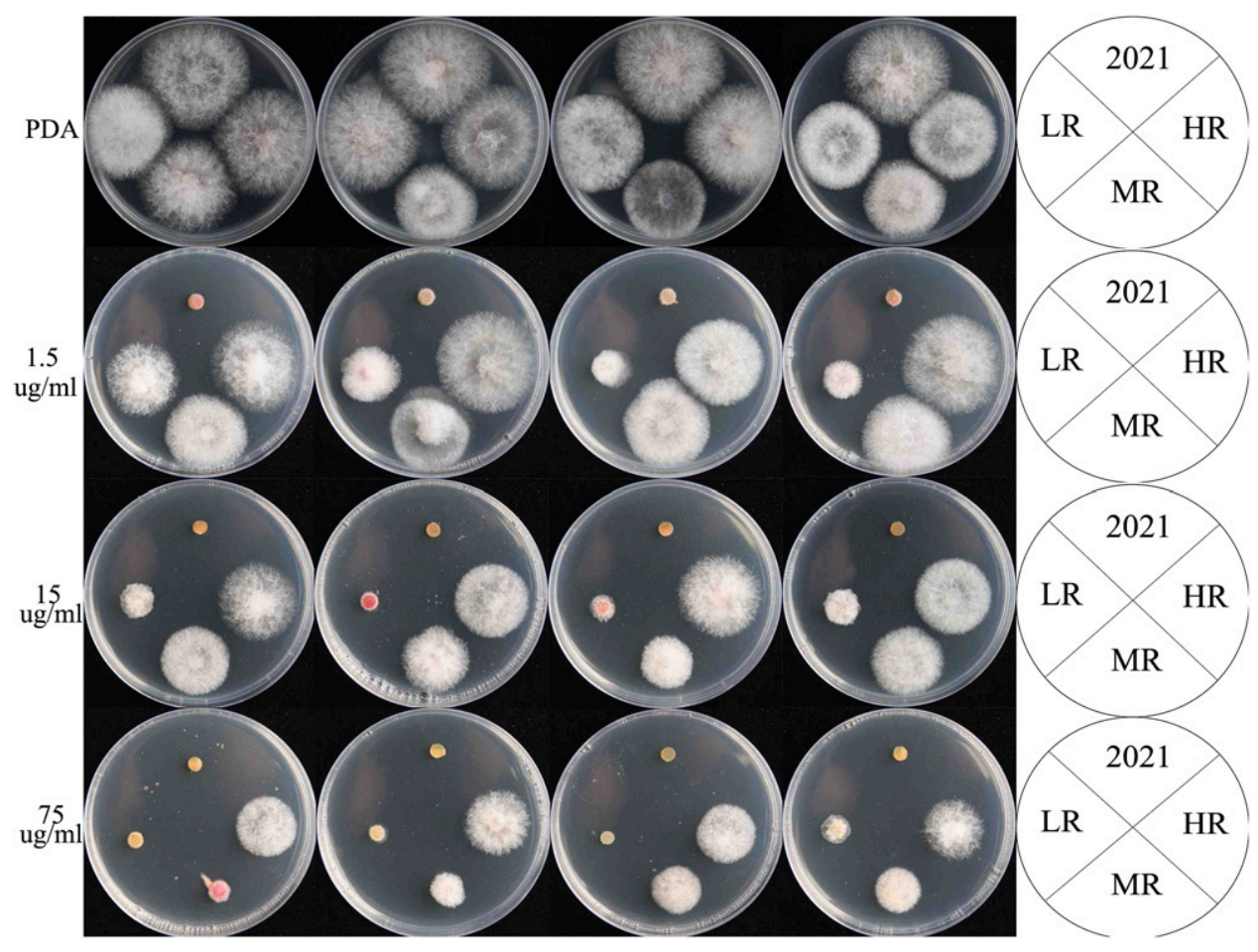

Fig. 1. Resistance stability of Fusarium asiaticum sensitive strain 2021 and several mutants with low resistance (LR), moderate resistance (MR), and high resistance (HR) on potato dextrose agar amended with phenamacril at $1.5,15$, or $75 \mu \mathrm{g} / \mathrm{ml}$.

Table 1. Frequency and 50\% effective concentration $\left(\mathrm{EC}_{50}\right)$ of phenamacril-resistant mutants from Fusarium asiaticum-sensitive strain 2021

\begin{tabular}{|c|c|c|c|c|c|}
\hline Level, codon ${ }^{\mathbf{a}}$ & Type $^{b}$ & $N^{\mathrm{c}}$ & Freq $(\%)^{\mathrm{d}}$ & Mutants & $\mathbf{E C}_{50}$ \\
\hline \multicolumn{6}{|l|}{ LR } \\
\hline 135 & $\mathrm{~A} \rightarrow \mathrm{T}$ & 14 & 17.1 & $\begin{array}{l}\text { T13, T26, T64, T66, T111, T2-23, T2-25, T2-26, T2-35, T2-38, T2-39, T2-51, } \\
\text { T2-75, T2-94 }\end{array}$ & $1.88-8.24$ \\
\hline 151 & $\mathrm{~V} \rightarrow \mathrm{M}$ & 1 & 1.2 & T93 & 2.17 \\
\hline 204 & $\mathrm{P} \rightarrow \mathrm{S}$ & 1 & 1.2 & $\mathrm{~T} 4$ & 1.48 \\
\hline 434 & $\mathrm{I} \rightarrow \mathrm{M}$ & 1 & 1.2 & $\mathrm{~T} 2-60$ & 2.95 \\
\hline 577 & $\mathrm{~A} \rightarrow \mathrm{T}$ & 1 & 1.2 & $\mathrm{~T} 76$ & 3.22 \\
\hline \multirow[t]{2}{*}{580} & $\mathrm{R} \rightarrow \mathrm{G}$ & 1 & 1.2 & $\mathrm{~T} 54$ & 12.41 \\
\hline & $\mathrm{R} \rightarrow \mathrm{H}$ & 1 & 1.2 & T86 & 2.53 \\
\hline 581 & $\mathrm{I} \rightarrow \mathrm{F}$ & 1 & 1.2 & $\mathrm{~T} 2-102$ & 3.45 \\
\hline \multicolumn{6}{|l|}{ MR } \\
\hline 418 & $S \rightarrow R$ & 1 & 1.2 & $\mathrm{~T} 40$ & 33.96 \\
\hline 424 & $\mathrm{I} \rightarrow \mathrm{R}$ & 2 & 2.4 & $\mathrm{~T} 2-89, \mathrm{~T} 2-56$ & $18.54-20.11$ \\
\hline 577 & $\mathrm{~A} \rightarrow \mathrm{G}$ & 3 & 3.7 & $\mathrm{~T} 21, \mathrm{~T} 2-14, \mathrm{~T} 2-76$ & $19.21-28.38$ \\
\hline \multicolumn{6}{|l|}{ HR } \\
\hline \multirow[t]{2}{*}{216} & $\mathrm{~K} \rightarrow \mathrm{R}$ & 1 & 1.2 & $\mathrm{~T} 79$ & 192.05 \\
\hline & $\mathrm{K} \rightarrow \mathrm{E}$ & 1 & 1.2 & T122 & 186.16 \\
\hline \multirow[t]{2}{*}{217} & $\mathrm{~S} \rightarrow \mathrm{P}$ & 2 & 2.4 & $\mathrm{~T} 105, \mathrm{~T} 2-5$ & $149.31-206.54$ \\
\hline & $\mathrm{S} \rightarrow \mathrm{L}$ & 27 & 32.9 & $\begin{array}{l}\text { T1, T3, T10, T12, T18, T25, T51, T91, T99, T106, T2-1, T2-7, T2-16, T2-18, } \\
\text { T2-29, T2-32, T2-37, T2-41, T2-48, T2-67, T2-69, T2-71, T2-85, T2-112, } \\
\text { T2-117, T2-125, T2-127 }\end{array}$ & $91.17-167.40$ \\
\hline \multirow[t]{3}{*}{420} & $\mathrm{E} \rightarrow \mathrm{K}$ & 22 & 26.8 & $\begin{array}{l}\text { T11, T14, T32, T48, T69, T73, T89, T95, T101, T102, T2-3, T2-9, T2-21, T2-61, } \\
\text { T2-78, T2-81, T2-82, T2-86, T2-90, T2-93, T2-98, T2-107 }\end{array}$ & $259.41-448.88$ \\
\hline & $\mathrm{E} \rightarrow \mathrm{G}$ & 1 & 1.2 & $\mathrm{~T} 50$ & 209.50 \\
\hline & $\mathrm{E} \rightarrow \mathrm{D}$ & 1 & 1.2 & $\mathrm{~T} 112$ & 232.18 \\
\hline
\end{tabular}

${ }^{a}$ Resistance level is determined by $\mathrm{EC}_{50}$ values: low resistance (LR, 1.5 to $15 \mu \mathrm{g} / \mathrm{ml}$ ), moderate resistance (MR, 15.1 to $75 \mu \mathrm{g} / \mathrm{ml}$ ), and high resistance $(\mathrm{HR},>75 \mu \mathrm{g} / \mathrm{ml})$. Codon = mutation position.

$\mathrm{b}$ Mutation types.

c Number of mutants; 82 mutants were randomly selected from the 239 resistant mutants.

${ }^{\mathrm{d}}$ Resistance frequency. 
Table 2. Mutation types, resistance level, and growth rate (GR) of the selected 82 resistant mutants ${ }^{\mathrm{a}}$

\begin{tabular}{|c|c|c|c|c|c|c|c|c|c|c|c|c|c|c|}
\hline Strains & Codon 135 & Codon 151 & Codon 204 & Codon 216 & Codon 217 & Codon 418 & Codon 420 & Codon 424 & Codon 434 & Codon 577 & Codon 580 & Codon 581 & $\begin{array}{c}\mathbf{E C}_{50} \\
(\mu \mathrm{g} / \mathrm{ml})\end{array}$ & $\begin{array}{c}\text { GR } \\
\text { (cm/day) }\end{array}$ \\
\hline 2021 & GCC (A) & GTG (V) & CCT (P) & AAG $(\mathrm{K})$ & TCA (S) & AGT (S) & GAA (E) & ATT (I) & $\operatorname{ATC}(\mathrm{I})$ & GCA (A) & CGT (R) & $\operatorname{ATC}(\mathrm{I})$ & 0.21 & $2.44 \pm 0.12$ \\
\hline $\mathrm{T} 13$ & $\operatorname{ACC}(\mathrm{T})$ & GTG (V) & CCT (P) & AAG (K) & TCA (S) & AGT (S) & GAA (E) & ATT (I) & $\operatorname{ATC}(\mathrm{I})$ & GCA (A) & CGT (R) & $\operatorname{ATC}(\mathrm{I})$ & 2.38 & $2.34 \pm 0.09$ \\
\hline $\mathrm{T} 26$ & $\operatorname{ACC}(\mathrm{T})$ & GTG (V) & CCT (P) & AAG (K) & TCA (S) & AGT (S) & GAA (E) & ATT (I) & $\operatorname{ATC}(\mathrm{I})$ & GCA (A) & CGT (R) & $\operatorname{ATC}(\mathrm{I})$ & 3.40 & $1.82 \pm 0.10$ \\
\hline T64 & $\operatorname{ACC}(\mathrm{T})$ & GTG (V) & CCT (P) & AAG $(\mathrm{K})$ & TCA (S) & AGT (S) & GAA (E) & ATT (I) & $\operatorname{ATC}(\mathrm{I})$ & GCA (A) & CGT (R) & $\operatorname{ATC}(\mathrm{I})$ & 2.52 & $2.17 \pm 0.04$ \\
\hline T66 & $\operatorname{ACC}(\mathrm{T})$ & GTG (V) & $\mathrm{CCT}(\mathrm{P})$ & AAG (K) & TCA (S) & AGT (S) & GAA (E) & ATT (I) & $\operatorname{ATC}(\mathrm{I})$ & GCA (A) & CGT (R) & $\operatorname{ATC}(\mathrm{I})$ & 2.86 & $1.07 \pm 0.06$ \\
\hline T111 & $\operatorname{ACC}(\mathrm{T})$ & GTG (V) & $\mathrm{CCT}(\mathrm{P})$ & AAG (K) & TCA (S) & AGT (S) & GAA (E) & ATT (I) & $\operatorname{ATC}(\mathrm{I})$ & GCA (A) & CGT (R) & $\operatorname{ATC}(\mathrm{I})$ & 5.23 & $1.08 \pm 0.13$ \\
\hline $\mathrm{T} 2-23$ & $\mathrm{ACC}(\mathrm{T})$ & GTG (V) & CCT (P) & AAG (K) & TCA (S) & AGT (S) & GAA (E) & ATT (I) & ATC (I) & GCA (A) & CGT (R) & $\operatorname{ATC}(\mathrm{I})$ & 4.06 & $2.06 \pm 0.03$ \\
\hline $\mathrm{T} 2-25$ & $\operatorname{ACC}(\mathrm{T})$ & GTG (V) & CCT (P) & AAG (K) & TCA (S) & AGT (S) & GAA (E) & ATT (I) & $\operatorname{ATC}(\mathrm{I})$ & GCA (A) & CGT (R) & $\operatorname{ATC}(\mathrm{I})$ & 2.55 & $2.32 \pm 0.05$ \\
\hline T2-26 & $\operatorname{ACC}(\mathrm{T})$ & GTG (V) & $\mathrm{CCT}(\mathrm{P})$ & AAG (K) & TCA (S) & AGT (S) & GAA (E) & ATT (I) & ATC (I) & GCA (A) & CGT (R) & $\operatorname{ATC}(\mathrm{I})$ & 3.05 & $1.41 \pm 0.09$ \\
\hline T2-35 & $\operatorname{ACC}(T)$ & GTG (V) & $\mathrm{CCT}(\mathrm{P})$ & AAG (K) & TCA (S) & AGT (S) & GAA (E) & ATT (I) & ATC (I) & GCA (A) & CGT (R) & ATC (I) & 1.88 & $2.47 \pm 0.10$ \\
\hline $\mathrm{T} 2-38$ & $\operatorname{ACC}(\mathrm{T})$ & GTG (V) & $\mathrm{CCT}(\mathrm{P})$ & AAG (K) & TCA (S) & AGT (S) & GAA (E) & ATT (I) & $\operatorname{ATC}(\mathrm{I})$ & GCA (A) & CGT (R) & $\operatorname{ATC}(\mathrm{I})$ & 8.24 & $1.29 \pm 0.05$ \\
\hline T2-39 & $\operatorname{ACC}(\mathrm{T})$ & GTG (V) & $\mathrm{CCT}(\mathrm{P})$ & AAG $(\mathrm{K})$ & TCA (S) & AGT (S) & GAA (E) & ATT (I) & $\operatorname{ATC}(\mathrm{I})$ & GCA (A) & CGT (R) & $\operatorname{ATC}(\mathrm{I})$ & 5.99 & $1.72 \pm 0.09$ \\
\hline $\mathrm{T} 2-51$ & $\operatorname{ACC}(\mathrm{T})$ & GTG (V) & $\mathrm{CCT}(\mathrm{P})$ & AAG $(\mathrm{K})$ & TCA (S) & AGT (S) & GAA (E) & ATT (I) & $\operatorname{ATC}(\mathrm{I})$ & GCA (A) & CGT (R) & $\operatorname{ATC}(\mathrm{I})$ & 4.06 & $2.66 \pm 0.02$ \\
\hline $\mathrm{T} 2-75$ & $\operatorname{ACC}(\mathrm{T})$ & GTG (V) & $\mathrm{CCT}(\mathrm{P})$ & AAG $(\mathrm{K})$ & TCA (S) & AGT (S) & GAA (E) & ATT (I) & $\operatorname{ATC}(\mathrm{I})$ & GCA (A) & CGT (R) & $\operatorname{ATC}(\mathrm{I})$ & 7.26 & $1.72 \pm 0.02$ \\
\hline T2-94 & $\operatorname{ACC}(\mathrm{T})$ & GTG (V) & CCT (P) & AAG $(\mathrm{K})$ & TCA (S) & AGT (S) & GAA (E) & ATT (I) & $\operatorname{ATC}(\mathrm{I})$ & GCA (A) & CGT (R) & $\operatorname{ATC}(\mathrm{I})$ & 2.57 & $1.69 \pm 0.07$ \\
\hline T93 & GCC (A) & ATG (M) & CCT (P) & AAG (K) & TCA (S) & AGT (S) & GAA (E) & ATT (I) & $\operatorname{ATC}(\mathrm{I})$ & GCA (A) & CGT (R) & ATC (I) & 2.17 & $2.66 \pm 0.05$ \\
\hline $\mathrm{T} 4$ & GCC (A) & GTG (V) & TCT (S) & AAG $(\mathrm{K})$ & TCA (S) & AGT (S) & GAA (E) & ATT (I) & $\operatorname{ATC}(\mathrm{I})$ & GCA (A) & CGT (R) & $\operatorname{ATC}(\mathrm{I})$ & 1.48 & $1.91 \pm 0.06$ \\
\hline T79 & GCC (A) & GTG (V) & CCT (P) & AGG (R) & TCA (S) & AGT (S) & GAA (E) & ATT (I) & $\operatorname{ATC}(\mathrm{I})$ & GCA (A) & CGT (R) & $\operatorname{ATC}(\mathrm{I})$ & 192.05 & $1.74 \pm 0.02$ \\
\hline T122 & GCC (A) & GTG (V) & CCT (P) & GAG (E) & TCA (S) & AGT (S) & GAA (E) & ATT (I) & ATC (I) & GCA (A) & CGT (R) & ATC (I) & 186.16 & $1.41 \pm 0.03$ \\
\hline T105 & GCC (A) & GTG (V) & CCT (P) & AAG (K) & $\mathrm{CCA}(\mathrm{P})$ & AGT (S) & GAA (E) & ATT (I) & $\operatorname{ATC}(\mathrm{I})$ & GCA (A) & CGT (R) & $\operatorname{ATC}(\mathrm{I})$ & 206.54 & $2.46 \pm 0.03$ \\
\hline T2-5 & GCC (A) & GTG (V) & CCT (P) & AAG (K) & $\mathrm{CCA}(\mathrm{P})$ & AGT (S) & GAA (E) & ATT (I) & $\operatorname{ATC}(\mathrm{I})$ & GCA (A) & CGT (R) & $\operatorname{ATC}(\mathrm{I})$ & 149.31 & $1.93 \pm 0.04$ \\
\hline $\mathrm{T} 1$ & GCC (A) & GTG (V) & CCT $(\mathrm{P})$ & AAG (K) & TTA (L) & AGT (S) & GAA (E) & ATT (I) & $\operatorname{ATC}(\mathrm{I})$ & GCA (A) & CGT (R) & $\operatorname{ATC}(\mathrm{I})$ & 109.75 & $2.07 \pm 0.12$ \\
\hline $\mathrm{T} 3$ & GCC (A) & GTG (V) & CCT (P) & AAG (K) & TTA (L) & AGT (S) & GAA (E) & ATT (I) & $\operatorname{ATC}(\mathrm{I})$ & GCA (A) & CGT (R) & ATC (I) & 150.96 & $1.99 \pm 0.06$ \\
\hline $\mathrm{T} 10$ & GCC (A) & GTG (V) & CCT (P) & AAG (K) & TTA (L) & AGT (S) & GAA (E) & ATT (I) & $\operatorname{ATC}(\mathrm{I})$ & GCA (A) & CGT (R) & $\operatorname{ATC}(\mathrm{I})$ & 126.71 & $2.51 \pm 0.02$ \\
\hline $\mathrm{T} 12$ & GCC (A) & GTG (V) & CCT (P) & AAG (K) & TTA (L) & AGT (S) & GAA (E) & ATT (I) & ATC (I) & GCA (A) & CGT (R) & ATC (I) & 156.29 & $1.62 \pm 0.07$ \\
\hline T18 & GCC (A) & GTG (V) & CCT (P) & AAG (K) & TTA (L) & AGT (S) & GAA & ATT (I) & (I) & GCA & CGT & $\operatorname{ATC}(\mathrm{I})$ & 119.07 & $2.47 \pm 0.04$ \\
\hline $\mathrm{T} 25$ & GCC (A) & GTG (V) & CCT $(\mathrm{P})$ & AAG (K) & TTA (L) & AGT (S) & G & (I) & I) & 4) & CGT & (I) & 36 & $1.87 \pm 0.01$ \\
\hline T51 & GCC (A) & GTG (V) & CCT $(\mathrm{P})$ & AAG (K) & TTA (L) & AGT (S) & G & (I) & (I) & $\mathrm{GC} A$ & CGT & (I) & 10 & $2.43 \pm 0.03$ \\
\hline T91 & GCC (A) & GTG (V) & CCT $(\mathrm{P})$ & AAG (K) & TTA (L) & AGT (S) & G & (I) & (I) & $\mathrm{GCA}$ & CGT & Al & 35 & $2.57 \pm 0.05$ \\
\hline T99 & GCC (A) & GTG (V) & CCT $(\mathrm{P})$ & AAG (K) & TTA (L) & AGT (S) & G & (I) & (I) & GCA & CGT & AT & 73 & $1.95 \pm 0.03$ \\
\hline T106 & GCC (A) & GTG (V) & CCT (P) & AAG (K) & TTA (L) & A & G & (I) & (I) & GCA & R) & $\operatorname{ATC}(\mathrm{I})$ & 167.40 & $1.28 \pm 0.04$ \\
\hline $\mathrm{T} 2-1$ & GCC (A) & GTG (V) & CCT (P) & AAG (K) & TTA (L) & AGT (S) & GAA (E) & ATT (I) & $\operatorname{ATC}(\mathrm{I})$ & GCA (A) & CGT (R) & $\operatorname{ATC}(\mathrm{I})$ & 105.85 & $2.31 \pm 0.07$ \\
\hline $\mathrm{T} 2-7$ & GCC (A) & GTG (V) & CCT (P) & AAG (K) & TTA (L) & AGT (S) & GAA (E) & ATT (I) & ATC (I) & GCA (A) & CGT (R) & ATC (I) & 98.95 & $2.38 \pm 0.08$ \\
\hline T2-16 & GCC (A) & GTG (V) & CCT (P) & AAG $(\mathrm{K})$ & TTA (L) & AGT (S) & GAA (E) & ATT (I) & $\operatorname{ATC}(\mathrm{I})$ & GCA (A) & CGT (R) & $\operatorname{ATC}(\mathrm{I})$ & 149.77 & $2.39 \pm 0.02$ \\
\hline T2-18 & GCC (A) & GTG (V) & CCT $(\mathrm{P})$ & AAG (K) & TTA (L) & AGT (S) & GAA (E) & ATT (I) & $\operatorname{ATC}(\mathrm{I})$ & GCA (A) & CGT (R) & $\operatorname{ATC}(\mathrm{I})$ & 125.65 & $1.32 \pm 0.02$ \\
\hline T2-29 & GCC (A) & GTG (V) & CCT (P) & AAG $(\mathrm{K})$ & TTA (L) & AGT (S) & GAA (E) & ATT (I) & $\operatorname{ATC}(\mathrm{I})$ & GCA (A) & CGT (R) & $\operatorname{ATC}(\mathrm{I})$ & 124.69 & $2.45 \pm 0.03$ \\
\hline T2-32 & GCC (A) & GTG (V) & CCT (P) & AAG $(\mathrm{K})$ & TTA (L) & AGT (S) & GAA (E) & ATT (I) & $\operatorname{ATC}(\mathrm{I})$ & GCA (A) & CGT (R) & $\operatorname{ATC}(\mathrm{I})$ & 106.36 & $0.57 \pm 0.05$ \\
\hline T2-37 & GCC (A) & GTG (V) & CCT (P) & AAG $(\mathrm{K})$ & TTA (L) & AGT (S) & GAA (E) & ATT (I) & $\operatorname{ATC}(\mathrm{I})$ & GCA (A) & CGT (R) & $\operatorname{ATC}(\mathrm{I})$ & 92.41 & $2.52 \pm 0.02$ \\
\hline T2-41 & GCC (A) & GTG (V) & CCT (P) & AAG $(\mathrm{K})$ & TTA (L) & AGT (S) & GAA (E) & ATT (I) & $\operatorname{ATC}(\mathrm{I})$ & GCA (A) & CGT (R) & $\operatorname{ATC}(\mathrm{I})$ & 91.17 & $2.07 \pm 0.01$ \\
\hline T2-48 & GCC (A) & GTG (V) & CCT (P) & AAG (K) & TTA (L) & AGT (S) & GAA (E) & ATT (I) & $\operatorname{ATC}(\mathrm{I})$ & GCA (A) & CGT (R) & ATC (I) & 95.34 & $2.44 \pm 0.05$ \\
\hline T2-67 & GCC (A) & GTG (V) & CCT (P) & AAG (K) & TTA (L) & AGT (S) & GAA (E) & ATT (I) & $\operatorname{ATC}(\mathrm{I})$ & GCA (A) & CGT (R) & ATC (I) & 98.95 & $2.22 \pm 0.02$ \\
\hline T2-69 & GCC (A) & GTG (V) & CCT (P) & AAG (K) & TTA (L) & AGT (S) & GAA (E) & ATT (I) & $\operatorname{ATC}(\mathrm{I})$ & GCA (A) & CGT (R) & $\operatorname{ATC}(\mathrm{I})$ & 124.68 & $2.62 \pm 0.02$ \\
\hline T2-71 & GCC (A) & GTG (V) & $\mathrm{CCT}(\mathrm{P})$ & AAG (K) & TTA (L) & AGT (S) & GAA (E) & ATT (I) & $\operatorname{ATC}(\mathrm{I})$ & GCA (A) & CGT (R) & $\operatorname{ATC}(\mathrm{I})$ & 140.49 & $2.20 \pm 0.10$ \\
\hline T2-85 & GCC (A) & GTG (V) & CCT (P) & AAG $(\mathrm{K})$ & TTA (L) & AGT (S) & GAA (E) & ATT (I) & ATC (I) & GCA (A) & CGT (R) & $\operatorname{ATC}(\mathrm{I})$ & 102.91 & $1.91 \pm 0.05$ \\
\hline $\mathrm{T} 2-112$ & GCC (A) & GTG (V) & CCT (P) & AAG $(\mathrm{K})$ & TTA (L) & AGT (S) & GAA (E) & ATT (I) & $\operatorname{ATC}(\mathrm{I})$ & GCA (A) & CGT (R) & $\operatorname{ATC}(\mathrm{I})$ & 118.91 & $2.45 \pm 0.03$ \\
\hline $\mathrm{T} 2-117$ & GCC (A) & GTG (V) & CCT $(\mathrm{P})$ & AAG (K) & TTA (L) & AGT (S) & GAA (E) & ATT (I) & $\operatorname{ATC}(\mathrm{I})$ & GCA (A) & CGT (R) & $\operatorname{ATC}(\mathrm{I})$ & 140.65 & $1.52 \pm 0.03$ \\
\hline $\mathrm{T} 2-125$ & GCC (A) & GTG (V) & CCT (P) & AAG $(\mathrm{K})$ & TTA (L) & AGT (S) & GAA (E) & ATT (I) & $\operatorname{ATC}(\mathrm{I})$ & GCA (A) & CGT (R) & $\operatorname{ATC}(\mathrm{I})$ & 125.86 & $1.92 \pm 0.02$ \\
\hline $\mathrm{T} 2-127$ & GCC (A) & GTG (V) & CCT $(\mathrm{P})$ & AAG (K) & TTA (L) & AGT (S) & GAA (E) & ATT (I) & $\operatorname{ATC}(\mathrm{I})$ & GCA (A) & CGT (R) & $\operatorname{ATC}(\mathrm{I})$ & 131.45 & $0.65 \pm 0.03$ \\
\hline $\mathrm{T} 40$ & GCC (A) & GTG (V) & CCT (P) & AAG $(\mathrm{K})$ & TCA (S) & AGG (R) & GAA (E) & ATT (I) & $\operatorname{ATC}(\mathrm{I})$ & GCA (A) & CGT (R) & $\operatorname{ATC}(\mathrm{I})$ & 33.96 & $2.21 \pm 0.07$ \\
\hline T11 & GCC (A) & GTG (V) & CCT (P) & AAG (K) & TCA (S) & AGT (S) & AAA (K) & ATT (I) & $\operatorname{ATC}(\mathrm{I})$ & GCA (A) & CGT (R) & $\operatorname{ATC}(\mathrm{I})$ & 431.03 & $1.33 \pm 0.11$ \\
\hline T14 & GCC (A) & GTG (V) & CCT (P) & AAG (K) & TCA (S) & AGT (S) & AAA (K) & ATT (I) & $\operatorname{ATC}(\mathrm{I})$ & GCA (A) & CGT (R) & $\operatorname{ATC}(\mathrm{I})$ & 308.31 & $1.71 \pm 0.12$ \\
\hline $\mathrm{T} 32$ & GCC (A) & GTG (V) & CCT $(\mathrm{P})$ & AAG (K) & TCA (S) & AGT (S) & AAA (K) & ATT (I) & $\operatorname{ATC}(\mathrm{I})$ & GCA (A) & CGT (R) & $\operatorname{ATC}(\mathrm{I})$ & 448.88 & $1.32 \pm 0.12$ \\
\hline T48 & GCC (A) & GTG (V) & CCT $(\mathrm{P})$ & AAG (K) & TCA (S) & AGT (S) & AAA (K) & ATT (I) & $\operatorname{ATC}(\mathrm{I})$ & GCA (A) & CGT (R) & $\operatorname{ATC}(\mathrm{I})$ & 320.16 & $0.61 \pm 0.01$ \\
\hline T69 & GCC (A) & GTG (V) & CCT $(\mathrm{P})$ & AAG (K) & TCA (S) & AGT (S) & AAA (K) & ATT (I) & $\operatorname{ATC}(\mathrm{I})$ & GCA (A) & CGT (R) & $\operatorname{ATC}(\mathrm{I})$ & 426.62 & $1.85 \pm 0.04$ \\
\hline $\mathrm{T} 73$ & GCC (A) & GTG (V) & CCT (P) & AAG (K) & TCA (S) & AGT (S) & AAA $(\mathrm{K})$ & ATT (I) & $\operatorname{ATC}(\mathrm{I})$ & GCA (A) & CGT (R) & $\operatorname{ATC}(\mathrm{I})$ & 330.09 & $1.26 \pm 0.12$ \\
\hline T89 & GCC (A) & GTG (V) & CCT (P) & AAG $(\mathrm{K})$ & TCA (S) & AGT (S) & AAA $(\mathrm{K})$ & ATT (I) & ATC (I) & GCA (A) & CGT (R) & ATC (I) & 293.84 & $1.79 \pm 0.02$ \\
\hline T95 & GCC (A) & GTG (V) & CCT (P) & AAG (K) & TCA (S) & AGT (S) & AAA $(\mathrm{K})$ & ATT (I) & $\operatorname{ATC}(\mathrm{I})$ & GCA (A) & CGT (R) & $\operatorname{ATC}(\mathrm{I})$ & 259.41 & $1.44 \pm 0.05$ \\
\hline T101 & GCC (A) & GTG (V) & $\mathrm{CCT}(\mathrm{P})$ & AAG $(\mathrm{K})$ & TCA (S) & AGT (S) & $\operatorname{AAA}(\mathrm{K})$ & ATT (I) & $\operatorname{ATC}(\mathrm{I})$ & GCA (A) & CGT (R) & ATC (I) & 320.38 & $1.43 \pm 0.07$ \\
\hline T102 & GCC (A) & GTG (V) & CCT $(\mathrm{P})$ & AAG (K) & TCA (S) & AGT (S) & AAA $(\mathrm{K})$ & ATT (I) & $\operatorname{ATC}(\mathrm{I})$ & GCA (A) & CGT (R) & $\operatorname{ATC}(\mathrm{I})$ & 301.36 & $1.54 \pm 0.05$ \\
\hline $\mathrm{T} 2-3$ & GCC (A) & GTG (V) & $\mathrm{CCT}(\mathrm{P})$ & AAG $(\mathrm{K})$ & TCA (S) & AGT (S) & $\operatorname{AAA}(\mathrm{K})$ & ATT (I) & $\operatorname{ATC}(\mathrm{I})$ & GCA (A) & CGT (R) & ATC (I) & 338.38 & $1.62 \pm 0.10$ \\
\hline T2-9 & GCC (A) & GTG (V) & CCT (P) & AAG $(\mathrm{K})$ & TCA (S) & AGT (S) & AAA (K) & ATT (I) & $\operatorname{ATC}(\mathrm{I})$ & GCA (A) & CGT (R) & ATC (I) & 319.86 & $1.06 \pm 0.09$ \\
\hline T2-21 & GCC (A) & GTG (V) & CCT (P) & AAG $(\mathrm{K})$ & TCA (S) & AGT (S) & AAA (K) & ATT (I) & ATC (I) & GCA (A) & CGT (R) & $\operatorname{ATC}(\mathrm{I})$ & 239.43 & $1.64 \pm 0.06$ \\
\hline T2-61 & GCC (A) & GTG (V) & CCT (P) & AAG $(\mathrm{K})$ & TCA (S) & AGT (S) & AAA (K) & ATT (I) & $\operatorname{ATC}(\mathrm{I})$ & GCA (A) & CGT (R) & $\operatorname{ATC}(\mathrm{I})$ & 332.09 & $1.15 \pm 0.03$ \\
\hline T2-78 & GCC (A) & GTG (V) & CCT $(\mathrm{P})$ & AAG $(\mathrm{K})$ & TCA (S) & AGT (S) & AAA $(\mathrm{K})$ & ATT (I) & $\operatorname{ATC}(\mathrm{I})$ & GCA (A) & CGT (R) & $\operatorname{ATC}(\mathrm{I})$ & 287.37 & $1.32 \pm 0.07$ \\
\hline $\mathrm{T} 2-81$ & GCC (A) & GTG (V) & CCT $(\mathrm{P})$ & AAG $(\mathrm{K})$ & TCA (S) & AGT (S) & AAA $(\mathrm{K})$ & ATT (I) & $\operatorname{ATC}(\mathrm{I})$ & GCA (A) & CGT (R) & $\operatorname{ATC}(\mathrm{I})$ & 309.46 & $1.55 \pm 0.03$ \\
\hline T2-82 & GCC (A) & GTG (V) & CCT $(\mathrm{P})$ & AAG $(\mathrm{K})$ & TCA (S) & AGT (S) & AAA $(\mathrm{K})$ & ATT (I) & $\operatorname{ATC}(\mathrm{I})$ & GCA (A) & CGT (R) & $\operatorname{ATC}(\mathrm{I})$ & 289.42 & $1.82 \pm 0.07$ \\
\hline T2-86 & GCC (A) & GTG (V) & $\mathrm{CCT}(\mathrm{P})$ & AAG (K) & TCA (S) & AGT (S) & AAA $(\mathrm{K})$ & ATT (I) & $\operatorname{ATC}(\mathrm{I})$ & GCA (A) & CGT (R) & $\operatorname{ATC}(\mathrm{I})$ & 301.25 & $1.34 \pm 0.05$ \\
\hline T2-90 & GCC (A) & GTG (V) & CCT (P) & AAG $(\mathrm{K})$ & TCA (S) & AGT (S) & AAA $(\mathrm{K})$ & ATT (I) & ATC (I) & GCA (A) & CGT (R) & $\operatorname{ATC}(\mathrm{I})$ & 286.37 & $1.44 \pm 0.05$ \\
\hline T2-93 & GCC (A) & STG (V) & CCT (P) & AAG $(\mathrm{K})$ & TCA (S) & AGT (S) & AAA $(\mathrm{K})$ & ATT (I) & $\operatorname{ATC}(\mathrm{I})$ & GCA (A) & CGT (R) & $\operatorname{ATC}(\mathrm{I})$ & 278.31 & $1.22 \pm 0.02$ \\
\hline T2-98 & GCC (A) & STG (V) & CCT $(\mathrm{P})$ & AAG (K) & TCA (S) & AGT (S) & AAA $(\mathrm{K})$ & ATT (I) & $\operatorname{ATC}(\mathrm{I})$ & GCA (A) & CGT (R) & $\operatorname{ATC}(\mathrm{I})$ & 269.94 & $0.82 \pm 0.02$ \\
\hline T2-107 & GCC (A) & GTG (V) & CCT (P) & AAG $(\mathrm{K})$ & TCA (S) & AGT (S) & AAA $(\mathrm{K})$ & ATT (I) & $\operatorname{ATC}(\mathrm{I})$ & GCA (A) & CGT (R) & $\operatorname{ATC}(\mathrm{I})$ & 269.36 & $1.52 \pm 0.02$ \\
\hline T50 & GCC (A) & GTG (V) & CCT $(\mathrm{P})$ & AAG (K) & TCA (S) & AGT (S) & GGA (G) & ATT (I) & $\operatorname{ATC}(\mathrm{I})$ & GCA (A) & CGT (R) & $\operatorname{ATC}(\mathrm{I})$ & 209.50 & $2.08 \pm 0.05$ \\
\hline T112 & GCC (A) & GTG (V) & CCT (P) & AAG $(\mathrm{K})$ & TCA (S) & AGT (S) & GAT (D) & ATT (I) & $\operatorname{ATC}(\mathrm{I})$ & GCA (A) & CGT (R) & $\operatorname{ATC}(\mathrm{I})$ & 232.18 & $2.00 \pm 0.07$ \\
\hline T2-89 & GCC (A) & GTG (V) & CCT (P) & AAG (K) & TCA (S) & AGT (S) & GAA (E) & $\operatorname{ACT}(\mathrm{T})$ & $\operatorname{ATC}(\mathrm{I})$ & GCA (A) & CGT (R) & ATC (I) & 20.11 & $2.03 \pm 0.03$ \\
\hline T2-56 & GCC (A) & GTG (V) & CCT (P) & AAG $(\mathrm{K})$ & TCA (S) & AGT (S) & GAA (E) & $\operatorname{ACT}(\mathrm{T})$ & $\operatorname{ATC}(\mathrm{I})$ & GCA (A) & CGT (R) & ATC (I) & 18.54 & $0.67 \pm 0.05$ \\
\hline $\mathrm{T} 2-60$ & GCC (A) & GTG (V) & CCT $(\mathrm{P})$ & AAG $(\mathrm{K})$ & TCA (S) & AGT (S) & GAA (E) & ATT (I) & ATG (M) & GCA (A) & CGT (R) & $\operatorname{ATC}(\mathrm{I})$ & 2.95 & $1.80 \pm 0.04$ \\
\hline $\mathrm{T} 76$ & GCC (A) & GTG (V) & CCT (P) & AAG (K) & TCA (S) & AGT (S) & GAA (E) & ATT (I) & $\operatorname{ATC}(\mathrm{I})$ & $\operatorname{ACA}(\mathrm{T})$ & CGT (R) & ATC (I) & 3.22 & $2.37 \pm 0.05$ \\
\hline $\mathrm{T} 21$ & GCC (A) & GTG (V) & CCT (P) & AAG (K) & TCA (S) & AGT (S) & GAA (E) & ATT (I) & $\operatorname{ATC}(\mathrm{I})$ & GGA (G) & CGT (R) & $\operatorname{ATC}(\mathrm{I})$ & 28.38 & $2.26 \pm 0.03$ \\
\hline T2-14 & GCC (A) & GTG (V) & CCT (P) & AAG (K) & TCA (S) & AGT (S) & GAA (E) & ATT (I) & ATC (I) & GGA (G) & CGT (R) & $\operatorname{ATC}(\mathrm{I})$ & 26.21 & $2.52 \pm 0.07$ \\
\hline T2-76 & GCC (A) & GTG (V) & $\mathrm{CCT}(\mathrm{P})$ & AAG (K) & TCA (S) & AGT (S) & GAA (E) & ATT (I) & $\operatorname{ATC}(\mathrm{I})$ & GGA (G) & CGT (R) & $\operatorname{ATC}(\mathrm{I})$ & 19.21 & $2.37 \pm 0.05$ \\
\hline T54 & GCC (A) & GTG (V) & CCT (P) & AAG $(\mathrm{K})$ & TCA (S) & AGT (S) & GAA (E) & ATT (I) & ATC (I) & GCA (A) & GGT (G) & $\operatorname{ATC}(\mathrm{I})$ & 12.41 & $2.35 \pm 0.04$ \\
\hline T86 & GCC (A) & GTG (V) & CCT (P) & AAG $(\mathrm{K})$ & TCA (S) & AGT (S) & GAA (E) & ATT (I) & $\operatorname{ATC}(\mathrm{I})$ & GCA (A) & CAT $(\mathrm{H})$ & $\operatorname{ATC}(\mathrm{I})$ & 2.53 & $2.57 \pm 0.05$ \\
\hline T2-102 & GCC (A) & GTG (V) & CCT $(\mathrm{P})$ & AAG (K) & TCA (S) & AGT (S) & GAA (E) & ATT (I) & ATC (I) & GCA (A) & CGT (R) & TTC (F) & 3.45 & $1.89 \pm 0.02$ \\
\hline
\end{tabular}

a Strains $=$ strains and mutants and $\mathrm{EC}_{50}=50 \%$ effective concentration. 
217 produced slightly different colonies but mutants with mutations at codon 420 had distinctive colony morphologies and significantly reduced hyphal growth rates (Fig. 2; Table 2). Most of the highly resistant mutants produced significant fewer conidia than the parental strain 2021 (Fig. 3).

Virulence assays of induced mutants at codons 135, 217, and 420. Because the frequency of mutations at codons 135, 217, and 420 was much higher than at others, we choose the mutants
T13 (with mutation at codon 135); T25 (with mutation at codon 217); and T32, T50, and T112 (with mutation at codon 420) to assay virulence. T32, T50, and T112 represent the change of the amino acid $\mathrm{E} \rightarrow \mathrm{K}, \mathrm{E} \rightarrow \mathrm{G}$, and $\mathrm{E} \rightarrow \mathrm{D}$, respectively. In addition, $\mathrm{T} 25$ represents the change of amino acid $\mathrm{S} \rightarrow \mathrm{L}$; we did not choose the change of amino acid $\mathrm{S} \rightarrow \mathrm{P}$ because the conidia production of T105 and T2-5 was too little to assay the virulence. Assays with sensitive strain 2021 and $\mathrm{dH}_{2} \mathrm{O}$ as the blank control were conducted. Stems started to turn

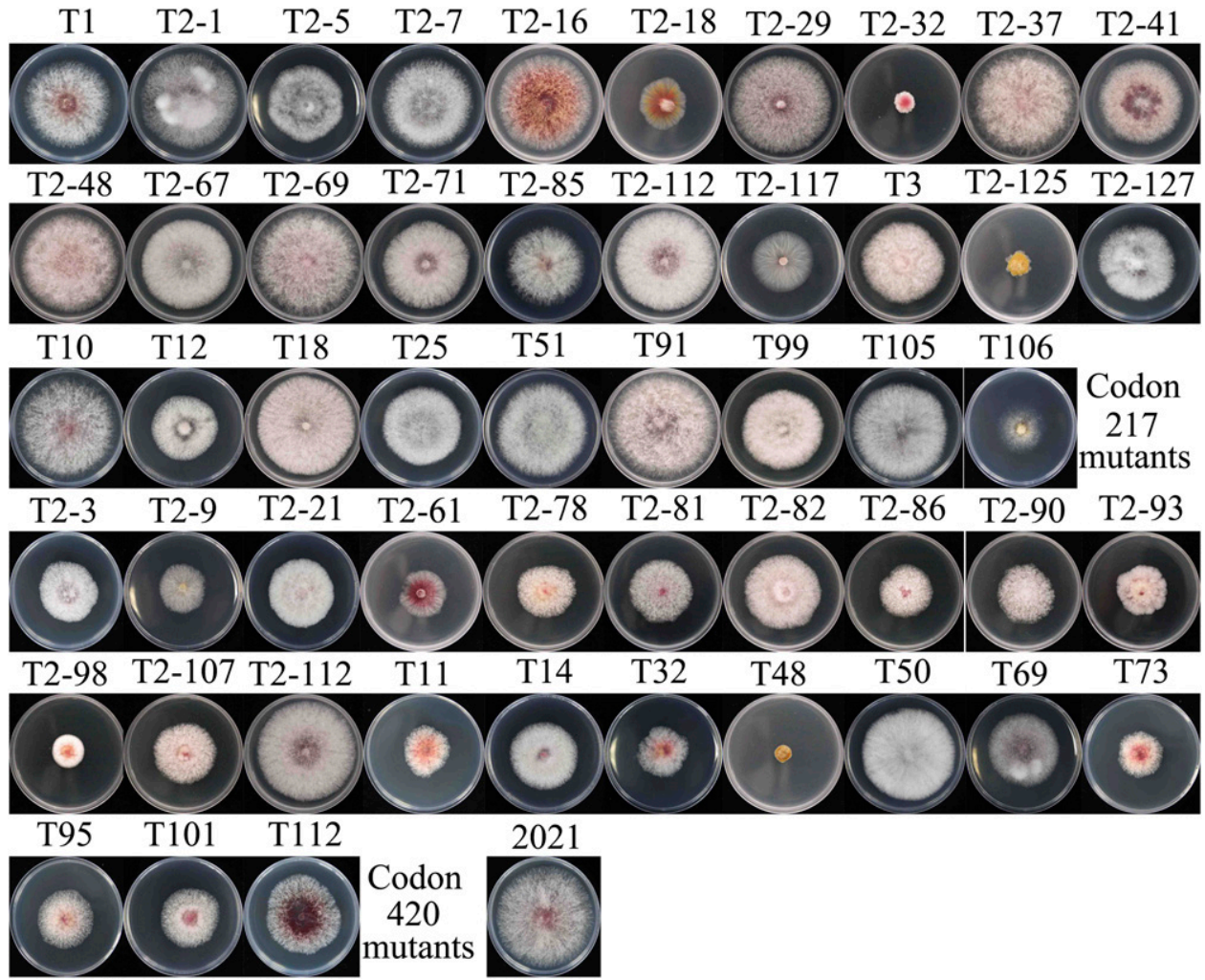

Fig. 2. Colony morphology of the Fusarium asiaticum-sensitive strain 2021 and the resistant mutants which have mutations at codon positions 217 and 420 . Mycelial plugs ( $5 \mathrm{~mm}$ in diameter) of $F$. asiaticum-sensitive strain 2021 and mutants were transferred to potato dextrose agar plates at $25^{\circ} \mathrm{C}$ for 3 days.

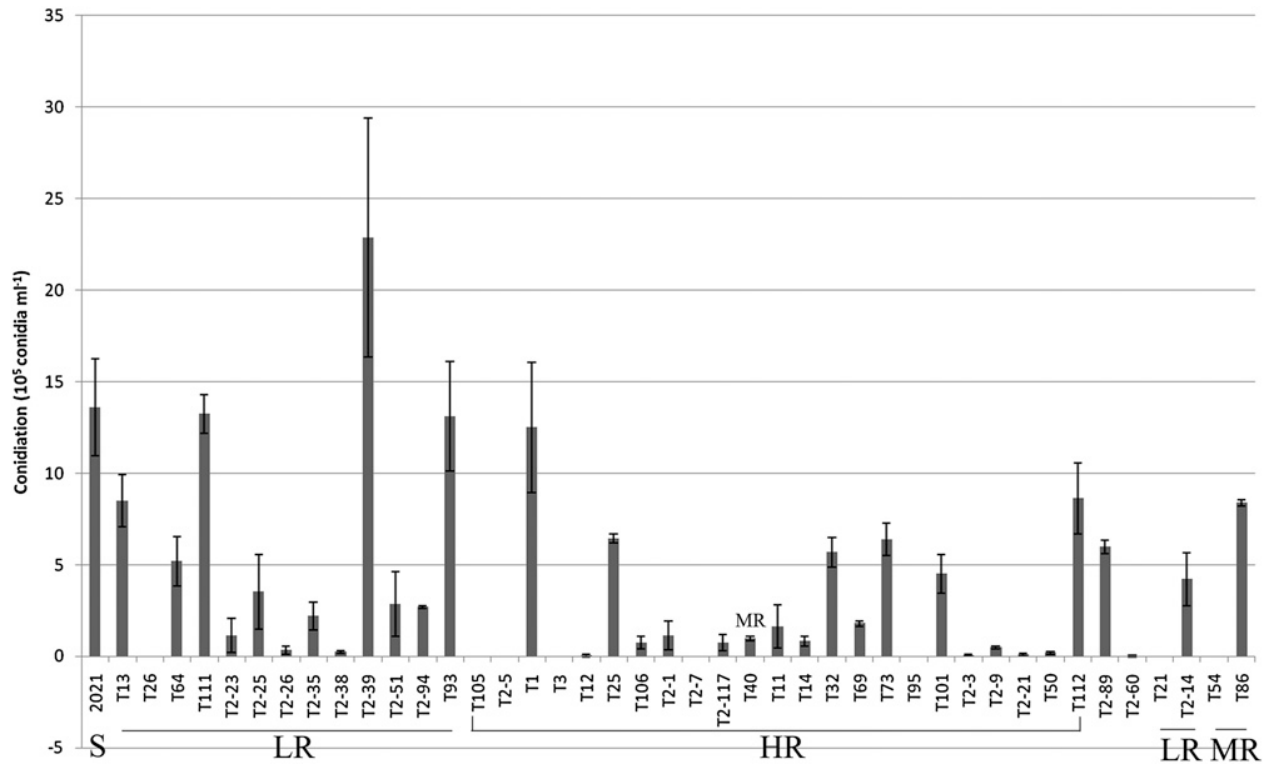

Fig. 3. Conidia production of randomly selected resistant mutants and the Fusarium asiaticum-sensitive strain 2021. All strains were represented by three flasks, and the flasks were incubated at $25^{\circ} \mathrm{C}$ for 7 days with shaking $(185 \mathrm{rpm})$. $\mathrm{LR}=$ low resistance, $\mathrm{MR}=$ moderate resistance, and $\mathrm{HR}=$ high resistance. 
brown within $3 \mathrm{dpi}$, and dark-brown lesions were evident on the coleoptiles of the inoculated seedlings by $7 \mathrm{dpi}$ (Fig. 4). The brown lesions of induced phenamacril-resistant mutants did not differ from the phenamacril-sensitive $F$. asiaticum strain 2021.

Homology modeling of $F$. asiaticum myosin5 motor domain. Because all of the mutations concentrated in the myosin5 motor domain, a three-dimensional model of the $F$. asiaticum myosin 5 motor domain was constructed (Fig. 5). Twelve codon positions of point mutations (codon 135, 151, 204, 216, 217, 418, 420, 424, 434, 577, 580, and 581) in the myosin5 motor domain conferring resistance to phenamacril were highlighted in the model (Fig. 5A). Mutations at amino acids 216, 217, and 420, which conferred high resistance to phenamacril, formed a similar "pocket" structure on the inside of the myosin5 motor domain. Because most mutations conferring high resistance occurred at codon positions 217 and 420, we called that the core region. Mutations located far from the core region were associated with lower resistance to phenamacril (Fig. 5B).

\section{Discussion}

Phenamacril is a novel cyanoacrylate fungicide that can strongly inhibit the mycelial growth of $F$. graminearum and exhibit an excellent efficacy in controlling FHB in the field (Li et al. 2008). In recent years, application of this fungicide for managing FHB has been extensively relied on. Although we have not found large numbers of isolates resistant to phenamacril in the fields, the selection pressure in the existence of this fungicide could not be ignored. To assess the potential risk in the development of resistance to phenamacril in $F$. asiaticum in the fields, we produced many mutants resistant to phenamacril in the laboratory. In this study, 1,000 mycelial plugs of the $F$. asiaticum-sensitive strain 2021 were used to produce resistant mutants on PDA amended with phenamacril at $10 \mu \mathrm{g} / \mathrm{ml}$. Of these sensitive mycelial plugs, 239 mutants resistant to phenamacril were obtained, with an overall phenamacril resistance frequency of 23.9\%. These mutants maintained their resistance through eight transfers on PDA plates for 40 days and when stored on PDA slants at $4{ }^{\circ} \mathrm{C}$ for 40 days. This stability indicated that the resistance to phenamacril was not a temporary adaptation of the pathogen to this fungicide.

Previous studies revealed that most phenamacril-resistant mutants exhibited similar mycelial growth rate when compared with sensitive strain 2021. However, some highly resistant mutants had distinctive colony morphologies. Several researchers found that gene deletion mutants would change colony morphology because the deletion strategy disrupted some gene functions (Farhat et al. 2013; Gu et al. 2015; Yu et al. 2014; Yun et al. 2015). However, distinctive colony morphologies of laboratory-induced $F$. asiaticum-resistant mutants might result from point mutations. Selection of fungicide led to the quick evolution of this fungus and changed some regions of the genome. Mutations in some regions directly conferred resistance whereas mutations in other regions directly or indirectly changed colony morphology. Conidia are responsible for the spread and infection of $F$. asiaticum during wheat flowering. However, most laboratory-induced HR mutants did not influence pathogenicity but sometimes reduced conidial production. This may reduce the fitness of the resistant mutants in the field and may slow down the formation of the resistant pathogen population for a few years.

Previous studies have reported that mutations at codons K216E, S217P, L, S418R, and E420G of myosin5 were responsible for resistance to the fungicide phenamacril (Zhang et al. 2015; Zheng et al. 2015). However, recent studies have confirmed that mutations in $m y$ osin 5 conferred resistance to phenamacril in the $F$. graminearum species complex. In our study, we found that mutations not only at codons $216,217,418$ and 420 of myosin 5 but also at codons 135 , 151, 204, 424, 434, 577, 580, and 581 of myosin 5 conferred resistance to phenamacril in F. asiaticum, and all of the mutations concentrated in the myosin 5 motor domain. In addition, we also found new point mutation types at codon positions K216R, E420K, and E420D. Among the selected 82 resistant mutants, the frequencies of HR, MR, and LR mutants were $67.1,7.3$, and $25.6 \%$, respectively. Interestingly, The quantity of mutants which had point mutations at codons 217 and 420 took up a large proportion (64.6\%) in resistant mutants; we named that the core region. According to our homology modeling of the $F$. asiaticum myosin 5 motor domain, we can see that mutations located far from the core region led to lower resistance to phenamacril. To our knowledge, this is the first report of an experiment studying phenamacril resistance by inducing $F$. asiaticum resistance in the laboratory and the point mutation in myosin 5 gene conferring resistance to phenamacril of laboratory-induced $F$. asiaticum populations.

Among resistant mutants in this study, different genotypes in $F$. asiaticum showed various resistance levels. We divided these mutations into two classes: one was resistance caused by target effect (HR) and the other was off-target effect (MR and LR). For the resistance mechanism of $\mathrm{HR}$, we can assume that mutations occurred in different amino acid residues which influence binding to phenamacril to different degrees. For example, site-directed mutagenesis of the P225, N230, and $\mathrm{H} 272$ residues of succinate dehydrogenase subunit B from Botrytis cinerea could influence the binding to carboxamide fungicides (Lalève et al. 2014). For MR and LR, we hypothesized that the different changes in the amino acid residue affected inordinately the expression level of the proteins FgFim and FgApsB, which can regulate the resistance level of phenamacril (Zheng et al. 2013, 2014). The exact mechanism of resistance to phenamacril needs to be explored further.

Resistance is encoded in the $F$. graminearum species complex genome and is thought to arise through the serial acquisition of point mutations in the myosin 5 gene. Resistance-associated mutations can serve as biomarkers that can be rapidly identified in the fields by PCR. Current molecular diagnostics amplify and detect known fungicide resistance-associated mutations, and their performance depends on the inclusion of a comprehensive catalog of these mutations. Although known mutations indicated larger resistance risk to phenamacril, phenamacril-resistant field isolates would not exhibit the same level of resistance and the same point mutation types. Large-scale monitoring for resistance to this fungicide should be performed, because field phenamacril resistance would help to clarify

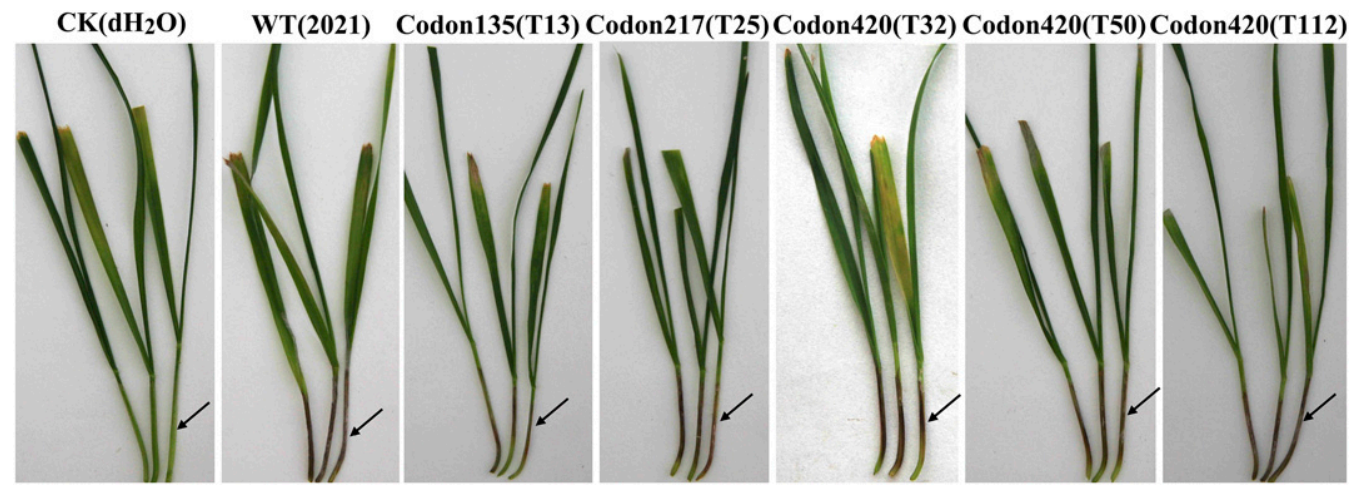

Fig. 4. Representative photographs of infected wheat coleoptiles inoculated with conidia of the Fusarium asiaticum-sensitive strain 2021 and mutants T13, T25, T32, T50, and T112. Brown lesions on the coleoptiles are indicated with arrows. $\mathrm{CK}=$ coleoptiles inoculated with distilled water. 
this point. Also, in order to delay the development of phenamacril resistance, tank-mixing the fungicide phenamacril with other fungicides will be effective in controlling FHB.

Our study showed the diversity within resistant mutations in myosin 5 and the genotype frequency of different drug-resistant mutants, as well as the resistance level of these mutations. The study provided a theoretical basis for assessing the JS399-19 risk of resistance, and the molecular technology for detecting and managing the resistance.

\section{Acknowledgments}

This work was supported by the National Science Foundation of China (31201543) and Special Fund for Agro-Scientific Research in the Public Interest (number 201303023).

\section{Literature Cited}

Bai, G., and Shaner, G. 2004. Management and resistance in wheat and barley to Fusarium head blight 1. Annu. Rev. Phytopathol. 42:135-161.
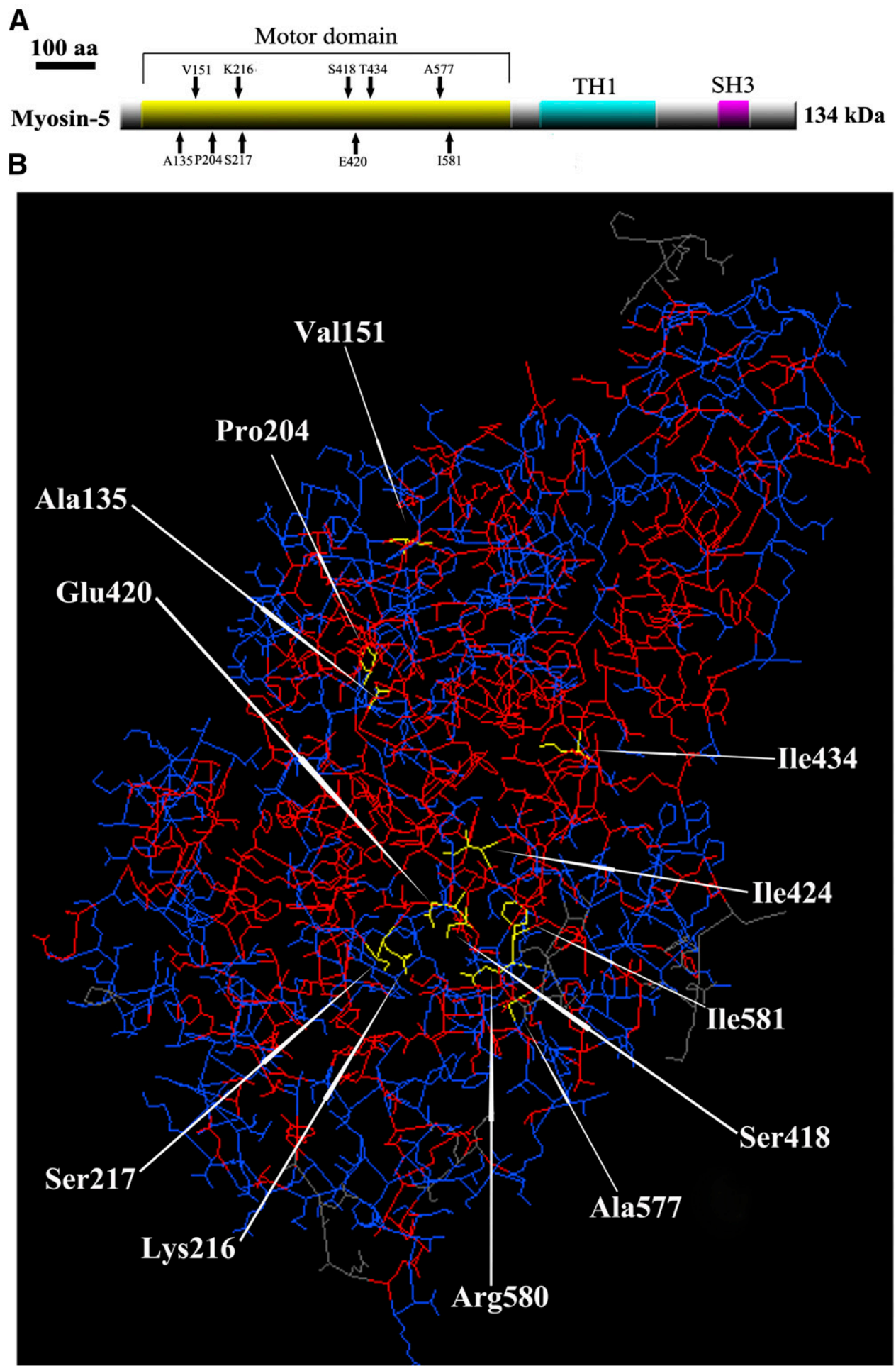

Fig. 5. Schematic representation of homology modeling of the Fusarium asiaticum myosin5 motor domain. A, Sites of Ala135, Val151, Pro204, Lys216, Ser217, Ser418, Glu420, lle424, lle434, Ala577, Arg580, and lle581 mutations are indicated with black arrowheads. The conserved motor domain, myosin tail homology 1 (TH1), and src homology domain 3 (SH3) are highlighted. Bar indicates 100 amino acids (aa). B, Amino acids Ala135, Val151, Pro204, Lys216, Ser217, Ser418, Glu420, lle424, lle434, Ala577, Arg580, and lle581 of the myosin5 motor domain are shown in yellow. Amino acids at these sites have been identified as mutations which conferred resistance in $F$. asiaticum. 
Bottalico, A. 1998. Fusarium diseases of cereals: Species complex and related mycotoxin profiles. Eur. J. Plant Pathol. 80:85-103.

Brandfass, C., and Karlovsky, P. 2008. Upscaled CTAB-based DNA extraction and real-time PCR assays for Fusarium culmorum and $F$. graminearum DNA in plant material with reduced sampling error. Int. J. Mol. Sci. 9:2306-2321.

Chen, L. F., Bai, G. H., and Desjardins, A. E. 2000. Recent advances in wheat head scab research in China. Pages 258-273 in: Proc. Int. Symp. Wheat Improvement for Scab Resistance. W. J. Raupp, Z. Ma, P. Chen, and D. Liu, eds. Nanjing Agricultural University, Jiangsu, China.

Chen, Y., Li, H., Chen, C., and Zhou, M. 2008. Sensitivity of Fusarium graminearum to fungicide JS399-19: In vitro determination of baseline sensitivity and the risk of developing fungicide resistance. Phytoparasitica 36:326-337.

Chen, Y., Wang, W.-X., Zhang, A.-F., Gu, C.-Y., Zhou, M.-G., and Gao, T.-C. 2011. Activity of the fungicide JS399-19 against Fusarium head blight of wheat and the risk of resistance. Agric. Sci. China 10:1906-1913.

Chen, Y., and Zhou, M. G. 2009. Characterization of Fusarium graminearum isolates resistant to both carbendazim and a new fungicide JS399-19. Phytopatho logy 99:441-446.

Diao, Y. M., Zhou, M. G., Wang, J. X., and Liu, D. R. 2012. Development of JS399-19-tebuconazole 48\% SC to control wheat scab. Agrochemicals 5:024.

Farhat, M. R., Shapiro, B. J., Kieser, K. J., Sultana, R., Jacobson, K. R., Victor, T. C., Warren, R. M., Streicher, E. M., Calver, A., Sloutsky, A., Kaur, D. Posey, J. E., Plikaytis, B., Oggioni, M. R., Gardy, J. L., Johnston, J. C., Rodrigues, M., Tang, P. K. C., Kato-Maeda, M., Borowsky, M. L., Muddukrishna, B., Kreiswirth, B. N., Kurepina, N., Galagan, J., Gagneux, S., Birren, B., Rubin, E. J., Lander, E. S., Sabeti, P. C., and Murray, M. 2013. Genomic analysis identifies targets of convergent positive selection in drugresistant Mycobacterium tuberculosis. Nat. Genet. 45:1183-1189.

Goswami, R. S., and Kistler, H. C. 2004. Heading for disaster: Fusarium graminearum on cereal crops. Mol. Plant Pathol. 5:515-525.

Gu, Q., Chen, Y., Liu, Y., Zhang, C., and Ma, Z. 2015. The transmembrane protein FgSho1 regulates fungal development and pathogenicity via the MAPK module Ste50-Ste11-Ste7 in Fusarium graminearum. New Phytol. 206:315-328.

Lalève, A., Gamet, S., Walker, A. S., Debieu, D., Toquin, V., and Fillinger, S. 2014. Site-directed mutagenesis of the P225, N230 and H272 residues of succinate dehydrogenase subunit B from Botrytis cinerea highlights different roles in enzyme activity and inhibitor binding. Environ. Microbiol. 16:2253-2266.

Li, H., Diao, Y., Wang, J., Chen, C., Ni, J., and Zhou, M. 2008. JS399-19, a new fungicide against wheat scab. Crop Prot. 27:90-95.

O’Donnell, K., Ward, T. J., Geiser, D. M., Kistler, H. C., and Aoki, T. 2004. Genealogical concordance between the mating type locus and seven other nuclear genes supports formal recognition of nine phylogenetically distinct species within the Fusarium graminearum clade. Fungal Genet. Biol. 41:600-623.

Parry, D. W., Jenkinson, P., and McLeod, L. 1995. Fusarium ear blight (scab) in small grain cereals-A review. Plant Pathol. 44:207-238.

Qiu, J., Xu, J., Yu, J., Bi, C., Chen, C., and Zhou, M. 2011. Localisation of the benzimidazole fungicide binding site of Gibberella zeae $\beta 2$-tubulin studied by site-directed mutagenesis. Pest Manage. Sci. 67:191-198.
Starkey, D. E., Ward, T. J., Aoki, T., Gale, L. R., Kistler, H. C., Geiser, D. M. Suga, H., Tóth, B., Varga, J., and O’Donnell, K. 2007. Global molecular surveillance reveals novel Fusarium head blight species and trichothecene toxin diversity. Fungal Genet. Biol. 44:1191-1204.

Suga, H., Karugia, G., Ward, T., Gale, L. R., Tomimura, K., Nakajima, T. Miyasaka, A., Koizumi, S., Kageyama, K., and Hyakumachi, M. 2008. Molecular characterization of the Fusarium graminearum species complex in Japan. Phytopathology 98:159-166.

Tóth, B., Mesterházy, A., Horváth, Z., Bartók, T., Varga, M., and Varga, J. 2005. Genetic variability of central European isolates of the Fusarium graminearum species complex. Eur. J. Plant Pathol. 113:35-45.

Windels, C. E. 2000. Economic and social impacts of Fusarium head blight: Changing farms and rural communities in the Northern Great Plains. Phyto pathology 90:17-21.

Wu, A. B., Li, H. P., Zhao, C. S., and Liao, Y. C. 2005. Comparative pathogenicity of Fusarium graminearum isolates from China revealed by wheat coleoptile and floret inoculations. Mycopathologia 160:75-83.

Yu, F., Gu, Q., Yun, Y., Yin, Y., Xu, J. R., Shim, W. B., and Ma, Z. 2014. The TOR signaling pathway regulates vegetative development and virulence in Fusarium graminearum. New Phytol. 203:219-232.

Yun, Y., Liu, Z., Yin, Y., Jiang, J., Chen, Y., Xu, J. R., and Ma, Z. 2015. Functional analysis of the Fusarium graminearum phosphatome. New Phytol. 207: 119-134.

Zhang, C., Chen, Y., Yin, Y., Ji, H. H., Shim, W. B., Hou, Y., Zhou, M., Li, X. d., and Ma, Z. 2015. A small molecule species specifically inhibits Fusarium myosin I. Environ. Microbiol. 17:2735-2746.

Zhang, D., Fan, F., Yang, J., Wang, X., Qiu, D., and Jiang, L. 2010a. FgTep1p is linked to the phosphatidylinositol-3 kinase signalling pathway and plays a role in the virulence of Fusarium graminearum on wheat. Mol. Plant Pathol. 11:495-502.

Zhang, J.-B., Li, H.-P., Dang, F.-J., Qu, B., Xu, Y.-B., Zhao, C.-S., and Liao, Y.-C 2007. Determination of the trichothecene mycotoxin chemotypes and associated geographical distribution and phylogenetic species of the Fusarium graminearum clade from China. Mycol. Res. 111:967-975.

Zhang, Y. J., Zhang, X. A., Chen, C. J., Zhou, M. G., and Wang, H. C. 2010b. Effects of fungicides JS399-19, azoxystrobin, tebuconazloe, and carbendazim on the physiological and biochemical indices and grain yield of winter wheat Pestic. Biochem. Physiol. 98:151-157.

Zheng, Z., Gao, T., Hou, Y., and Zhou, M. 2013. Involvement of the anucleate primary sterigmata protein $\mathrm{FgApsB}$ in vegetative differentiation, asexual development, nuclear migration, and virulence in Fusarium graminearum. FEMS Microbiol. Lett. 349:88-98.

Zheng, Z., Gao, T., Zhang, Y., Hou, Y., Wang, J., and Zhou, M. 2014. FgFim, a key protein regulating resistance to the fungicide JS399-19, asexual and sexual development, stress responses and virulence in Fusarium graminearum. Mol. Plant Pathol. 15:488-499.

Zheng, Z., Hou, Y., Cai, Y., Zhang, Y., Li, Y., and Zhou, M. 2015. Whole-genome sequencing reveals that mutations in myosin-5 confer resistance to the fungicide phenamacril in Fusarium graminearum. Sci. Rep. 5: Article 8248. doi:10.1038/ srep08248 